\title{
La experiencia AICLE del alumnado de primero de educación secundaria desde una perspectiva transicional
}

\author{
The CLIL experience of students belonging to the First Year of Secondary \\ Education from a transitional perspective
}

\begin{abstract}
A experiência CLIL dos alunos do primeiro ano do ensino secundário de uma perspectiva de transição

Natacha Rita Díaz Luis

Universidad de La Laguna, España alu0100815085@ull.edu.es https://orcid.org/0000-0002-9838-369X
\end{abstract}

\section{Resumen}

La metodología Aprendizaje Integrado de Contenidos en Lenguas Extranjeras (AICLE) ha adquirido gran relevancia en la educación no universitaria y su propagación en la región canaria (España) ha sido considerable durante este siglo a través de diversos programas de adaptación de enseñanza de lengua y contenidos que se ofrecen de forma simultánea. Por tal motivo, el objetivo de la presente investigación fue conocer la percepción de 50 alumnos del primer año de educación secundaria obligatoria de una institución localizada en el área metropolitana de la isla de Tenerife (España) en torno al uso de la metodología AICLE como estrategia educativa para favorecer la competencia comunicativa en lengua extranjera. Para ello, se elaboró una encuesta de naturaleza cuantitativa y cualitativa conformada por 15 ítems. Los resultados demuestran la necesidad de fortalecer ciertas dimensiones de la metodología AICLE relacionadas con el grado motivacional, la figura del auxiliar de conversación y la implementación de estrategias y recursos de apoyo, elementos esenciales para impulsar la formación en una lengua extranjera. Por ello, se puede recomendar un mayor seguimiento del alumnado que se encuentra en ese periodo de transición educativa, ya que pueden verse afectados tanto por el cambio del contexto educativo como por el aprendizaje mediante una lengua extranjera. 
Palabras clave: dificultad en el aprendizaje, educación bilingüe, enseñanza de idiomas, transición educativa.

\section{Abstract}

The CLIL methodology has acquired a great impact upon non-university education. Its proliferation in the Canary Islands (Spain) has been considerable during this century by means of transitional programs that aims to introduce the simultaneous learning of language and contents. This study aimed to analyze how the different areas such as sources, strategies, parental and academic support, the motivation, etc. take part in the bilingual program and how they contribute to the learning process of students. Firstly, a theoretical approach will be developed taking into account issues such as the importance of getting to know the consequences caused by moving from one educational stage to a different one and the gradual introduction and development of the CLIL methodology in the Canary Islands. Afterwards, 50 students belonging to the first year of Secondary Education and inserted in the CLIL methodology have been selected. A questionnaire has been utilized as an evaluative instrument that goaled to detect those areas that require to be revised and improved in the bilingual program. The different areas created for result analysis are the following: use of materials and resources regarding CLIL methodology, utilization of the foreign language and mother language, use of other different methodologies within CLIL, evaluation, degree of motivation and reflection upon the experience. These results have demonstrated the need to combine the different elements that are applied in the academic activity in a bilingual environment. It has been also resulted the necessity to revise the selection of human resources and to generate more professional training regarding the utilization of CLIL methodology. Above all, the results have revealed the great relevance and necessity to make a gradual study of pupils who are starting Secondary Education because of the dual handicap they may suffer: change in the educative context and the learning through a foreign language.

Keywords: foreign language teaching, bilingual education, educational transition, learning difficulties. 


\section{Resumo}

A metodologia de Aprendizagem Integrada de Conteúdos em Línguas Estrangeiras (CLIL) adquiriu grande relevância no ensino não universitário e a sua difusão na região das Canárias (Espanha) foi considerável durante este século através de vários programas de adaptação de ensino de línguas e conteúdos oferecidos em simultâneo. Por este motivo, o objetivo desta pesquisa foi conhecer a percepção de 50 alunos do primeiro ano do ensino médio obrigatório de uma instituição localizada na área metropolitana da ilha de Tenerife (Espanha) sobre a utilização da metodologia CLIL como estratégia educacional para promover a competência comunicativa em língua estrangeira. Para tanto, foi elaborada uma pesquisa quantitativa e qualitativa, composta por 15 itens. Os resultados demonstram a necessidade de reforçar certas dimensões da metodologia CLIL relacionadas com o grau motivacional, a figura do assistente de conversação e a implementação de estratégias e recursos de apoio, elementos essenciais para promover a formação em língua estrangeira. Por esse motivo, um maior acompanhamento dos alunos que se encontram nesse período de transição educacional pode ser recomendado, uma vez que eles podem ser afetados tanto pela mudança no contexto educacional quanto pelo aprendizado de uma língua estrangeira.

Palavras-chave: dificuldades de aprendizagem, educação bilíngue, ensino de línguas, transição educacional.

Fecha Recepción: Julio 2020

Fecha Aceptación: Febrero 2021

\section{Introducción}

El aprendizaje de la lengua extranjera (inglés) se ha convertido en un imperativo en materia comunicativa debido a la necesidad de conocer y dominar un medio de comunicación internacional. Este concepto del inglés como lingua franca ha promovido en numerosos centros europeos el crecimiento de la educación bilingüe (Comisión Europea, 2001), la cual se promueve desde una visión dual: como fin y como medio en el proceso de aprendizaje. Lengua y contenidos se fusionan en el sistema educativo para favorecer el aprendizaje de la lengua extranjera. Por eso, en el presente artículo se tratará de analizar el contexto de aprendizaje del alumnado perteneciente al primer año de educación secundaria obligatoria (ESO) en un programa de educación bilingüe.

Este primer curso se caracteriza, sin lugar a duda, por el surgimiento de nuevos cambios y exigencias que el alumno debe encarar, los cuales le pueden generar numerosos problemas que pueden llegar a afectar su trayectoria académica (Fernández, Mena y 
Riviere, 2010; Roca, 2010). Por eso, Monarca, Rappoport y Fernández González (2012) señalan que alrededor de $33 \%$ de los estudiantes se encuentran en una condición vulnerable sobre su rendimiento en este periodo de variación hacia la educación secundaria.

A medida que el alumnado avanza en los distintos cursos y etapas educativas, se observa una adquisición de la lengua más analítica en el afianzamiento de las destrezas y habilidades lingüísticas y cognitivas (Loaiza Villalba y Galindo Martínez, 2014; Signoret, 2013). Desde una perspectiva madurativa, el diseño de los programas bilingües está determinado por esta cuestión cognitiva, de ahí que la implementación de la educación bilingüe requiera de una perspectiva progresiva que permita adecuar los contenidos a la evolución cognitiva del alumnado. Para ello, sin embargo, se debe tener en cuenta que en la etapa de educación primaria un docente se encarga de la enseñanza y aprendizaje de diversas materias, mientras que en la educación secundaria cada docente imparte la materia de su especialidad. En este formato educativo cuyo profesorado está más fragmentado es necesario un mayor grado de cooperación y coordinación entre docentes (Julián de Vega, 2013), así como una mejor formación.

Esto último se evidencia en un estudio de Durán Martínez (2018), quien observó una mayor formación de la L2 en el profesorado de educación secundaria, en comparación con los docentes de la etapa anterior, los cuales contaban con una mejor formación metodológica en el ejercicio docente de programas bilingües.

Ahora bien, desde una perspectiva histórica, las islas Canarias (España) se han caracterizado por formularse como puerto franco entre diversas culturas donde se desarrollan diversas actividades comerciales. De hecho, su posición geográfica estratégica —entre los continentes africano, americano y europeo- ha sido un factor decisivo para que la lengua inglesa influya en el dialecto canario ${ }^{1}$. A partir del año 2003, la Comisión Europea dictaminó un plan para promover el aprendizaje de lenguas extranjeras entre ciudadanos europeos. Mediante la implementación de este plan, se han elaborado propuestas para que las distintas instituciones nacionales, regionales y locales promuevan el aprendizaje de idiomas.

En tal sentido, Frigols y Marsh (2014) analizaron el comienzo de programas de educación bilingüe (a partir del curso 2004-2005) en nueve centros de educación primaria de las islas Canarias. Si bien su implementación ha sido paulatina, se puede indicar que

\footnotetext{
${ }^{1}$ Vocablos tales como queque, piche o tifa, comúnmente empleados por los hablantes del dialecto canario, proceden del inglés cake, pitch y theft act, respectivamente, entre algunos anglicismos en dicho dialecto.
} 


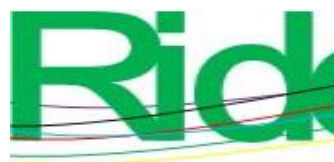

Revista Iberoamericana para la

Investigación y el Desarrollo Educativo

ISSN 2007 - 7467

dicha iniciativa ha presentado los resultados más altos en lo que se refiere a la exposición de la lengua extranjera, por lo que se ha convertido en una experiencia positiva para el alumnado perteneciente a ese programa educativo (Rodríguez-Sabiote, Madrid, OrtegaMartín y Hughes, 2018).

Posteriormente, en junio de 2005 este programa continuó con la incorporación de la metodología Aprendizaje Integrado de Contenidos en Lenguas Extranjeras (AICLE) en centros de educación secundaria obligatoria mediante el Proyecto de Secciones Bilingües (Martín Frigols y Marsh, 2014). Luego, en el curso 2006- 2007, la Consejería de Educación, Cultura y Deportes de la región canaria determinó la propagación de esta metodología simultánea de lengua y contenidos $-\mathrm{y}$ posteriormente cognición (Coyle, Hood y Marsh, 2010) — en centros de educación infantil, primaria y secundaria para favorecer la competencia comunicativa en la lengua extranjera, por lo que se empleó el Marco Común Europeo de Referencia para las Lenguas como fundamentación lingüística. Para la participación en tal programa, se indicaron requisitos tales como un mínimo de dos áreas no lingüísticas participantes, compromiso entre el claustro y el departamento de inglés para el desarrollo de esta metodología, un nivel B1 del profesorado en aquellas materias no lingüísticas que desearan ser parte del programa, así como la participación del profesorado en procesos de formación continua y en la elaboración de recursos acordes con la metodología AICLE.

No obstante, cabe destacar que el éxito de dichos programas bilingües puede verse altamente condicionado por el grado de estudio y reflexión sobre las prácticas en ese modelo educativo. Por tanto, existe la necesidad de profundizar en la efectividad de programas AICLE, los cuales deben atender las características del alumnado para promover en ellos un aprendizaje exitoso (Dalton-Puffer, Llinares, Lorenzo y Nikula, 2014), pues el doble enfoque en el aprendizaje que permite adquirir diversos objetivos al unísono de contenidos y lenguas extranjeras se ha visto favorecido por la investigación educativa y las prácticas multidisciplinares (Mehisto, 2012) ${ }^{2}$.

No obstante, durante el curso 2009-2010, Martín y Frigols (2014) observaron una gran evolución en los programas educativos bilingües de esta comunidad, lo cual se evidenció en el aumento de centros educativos participantes (169), la formación del perfil docente AICLE, la promulgación del uso de la plataforma Moodle y el incremento en la formación inicial y continua del profesorado participante (estancias en el extranjero, intercambios con docentes procedentes de países de habla inglesa, cursos de

\footnotetext{
2 A este doble foco, Coyle et al. (2010) han añadido un tercero: la capacidad y prácticas de cognición del alumnado, entendidas como habilidades de pensamiento.
} 
especialización e innovación metodológica). Además, a partir de este curso académico, se planteó un sistema de seguimiento y evaluación que debía tomar en cuenta los siguientes aspectos:

- Presentar una evaluación trimestral de los aspectos más importantes desarrollados en la CCP.

- Asistir a sesiones de seguimiento organizadas por la Dirección General de Ordenación e Innovación Educativa (DGOIE).

- Colaborar con el seguimiento de la DGOIE para la determinación del alcance de los resultados y avances obtenidos.

- Participar y hacer llegar a otros un cuestionario de satisfacción a la comunidad educativa (Martín y Frigols, 2014).

Este conjunto de parámetros que tratan de analizar el funcionamiento y la evolución de los programas de educación bilingüe se centran en la figura docente. En un periodo de inicio en la implementación de la metodología AICLE en centros de distintas etapas educativas, se requiere conocer la perspectiva del alumnado, esto es, su grado de satisfacción, los materiales o recursos que comúnmente emplea, su nueva visión de la lengua extranjera, las áreas que le suponen un mayor esfuerzo por la conjugación de lenguas, contenidos y cognición, entre otros.

El Plan de Impulso de las Lenguas Extranjera (Plan PILE), actualmente desarrollado en las islas Canarias, tiene como objetivo acoger etapas educativas no universitarias (educación infantil, primaria, secundaria, bachillerato, formación profesional y escuelas oficiales de idiomas) a través de centros públicos canarios. Este trata de otorgarle una nueva perspectiva al aprendizaje del inglés como fuente de conocimiento y medio de comunicación para el ámbito profesional, pues con ello se busca favorecer acciones como la empleabilidad y la movilidad de los individuos. Dicho plan, fragmentado en tres fases para su adaptación y evolución en la educación no universitaria canaria, actualmente se encuentra en la segunda fase de implantación. El objetivo final de este plan es que a partir del curso 2037-38, $40 \%$ del currículo de educación infantil y primaria, y $30 \%$ del currículo de educación secundaria se expongan en la L2.

El Plan PILE no solo se formula desde una perspectiva cuantitativa mediante el aumento del número de horas de exposición de la lengua extranjera mediante su utilización como vehículo de aprendizaje en materias no lingüísticas, pues su aplicación también demanda una implementación cualitativa referente al empleo de metodologías compatibles con este formato de aprendizaje, así como la formación inicial y continua del 


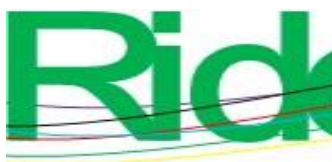

Revista Iberoamericana para la Investigación y el Desarrollo Educativo ISSN 2007 - 7467

profesorado de materias lingüísticas y no lingüísticas, y la actualización de los recursos de aprendizaje basados en contextos digitales.

A través del Plan PILE se observa una mayor insistencia en el seguimiento y evaluación continua del programa educativo, creando una comisión de carácter evaluativa, seguimiento en la formación del profesorado involucrado en el programa, plan de propuesta sobre el estudio de las áreas avanzadas más difíciles, y propuestas de mejora sobre el cumplimiento de las directrices y el grado de adquisición de la competencia comunicativa en lengua extranjera, entre los aspectos más relevantes. En resumen, el plan PILE establece los siguientes parámetros en el informe evaluativo:

1. El número de alumnado que consigue su acreditación linguiística en lenguas extranjeras en A2, B1 y B2.

2. El número de profesorado participante en las diferentes modalidades de formación para la mejora de la competencia comunicativa.

3. El número de profesorado participante en las diferentes modalidades de formación para la mejora metodológica en lenguas extranjeras.

4. El número de profesorado con acreditación lingüística que posibilite la impartición de los proyectos linguísticos en los centros educativos de Canarias.

5. El número de plazas docentes creadas a lo largo del desarrollo del plan y el cubrimiento de estas por el profesorado con diferentes perfiles administrativos.

6. El número de profesorado acreditado en B2 en lenguas extranjeras.

7. El número de centros que participen en proyectos europeos, internacionales u otros dentro de sus proyectos lingüísticos.

8. El número de centros que impartan por niveles al menos un tercio de su currículo en lengua inglesa.

9. El número de alumnado que accede a la doble titulación en bachillerato.

10. La implantación del modelo de enseñanza y de aprendizaje en lenguas extranjeras por distritos educativos.

11. El número de materiales creados por los centros educativos y su difusión.

En los distintos parámetros seleccionados por este plan, se puede señalar que se centra en la figura docente: el número de docentes participantes, sus respectivas acreditaciones lingüísticas, número de plazas, número de docentes con el nivel lingüístico específico B2, así como referencias sobre el centro educativo tales como el material creado por el centro, número de centros participantes en proyectos internacionales y la 
implementación de esta metodología por zonas educativas. La evaluación continua sobre los requisitos y la práctica de los docentes es de naturaleza incuestionable, pues son quienes diseñan estrategias de enseñanza-aprendizaje según la metodología AICLE, seleccionan, crean y comparten materiales y recursos ajustados a esta metodología, e impulsan la participación del alumnado en proyectos de carácter internacional, entre los aspectos más relevantes. No obstante, la finalidad de la metodología recae especialmente en el alumnado: su grado de adquisición de la competencia comunicativa en la lengua extranjera y su simultaneidad del aprendizaje de lenguas, contenidos y cognición.

\section{Metodología}

El objetivo de la presente investigación fue conocer la percepción del alumnado del primer año de educación secundaria obligatoria en torno al uso de la metodología AICLE como estrategia educativa para favorecer la competencia comunicativa en lengua extranjera.

Como se indicó en la introducción, la implementación de la educación bilingüe en las islas Canarias inició en el curso 2004-2005, desde un formato experimental en nueve centros educativos de educación infantil y primaria, y luego — un año más tarde — se incorporó en centros de educación secundaria ${ }^{3}$.

Ahora bien, el centro educativo escogido para el desarrollo de este estudio fue uno de los pioneros en aplicar dicha metodología de aprendizaje. Se trata de una institución localizada en el área metropolitana de la isla de Tenerife. Es un centro de índole pública, caracterizado por la utilización de metodologías innovadoras y por contar con un profesorado con una gran formación.

En este estudio participaron alumnos del primer año de educación secundaria obligatoria $(n=50)$, cuyas edades oscilaban entre los 11 y los 12 años. Más de $90 \%$ de este alumnado estudiaba en un centro público de educación primaria que empleaban la metodología AICLE en diversas asignaturas no lingüísticas. Este alumnado pertenecía al programa bilingüe desarrollado por el centro adaptado a partir de directrices del Plan PILE. El sistema de selección del alumnado perteneciente a este programa se basó en los resultados obtenidos en la materia de Primera Lengua Extranjera (Inglés) y el resto en asignaturas no lingüísticas que se han ejercido mediante la metodología AICLE en el último curso de educación primaria.

\footnotetext{
$3 \mathrm{Su}$ introducción en centros de educación secundaria también se desarrolla desde una perspectiva experimental y observacional.
} 
Para la realización de este estudio se empleó una encuesta de naturaleza cuantitativa y cualitativa enfocada en el alumnado. Los ítems que conformaron dicha encuesta (un total de 15 se enfocaron en aspectos vinculados con la implementación de la metodología AICLE, como la utilización de la lengua extranjera en el aula, la utilización de recursos para el estudio de contenidos, lengua y cognición, la figura del auxiliar de conversación como recurso humano en el proceso de aprendizaje, la conjugación de la metodología AICLE mediante otras metodologías como el aprendizaje basado en proyectos y el aprendizaje cooperativo. Mediante una actividad de autorreflexión, el alumnado debía determinar el grado de cumplimiento de las afirmaciones propuestas a través de cuatro parámetros: nunca, a veces, generalmente y siempre. En algunas de las afirmaciones planteadas se solicitó la justificación de la respuesta ofrecida para recabar más información sobre su experiencia en el primer año de educación secundaria. Las distintas dimensiones seleccionadas para esta investigación fueron las siguientes, tal y como se observa en la siguiente tabla:

Tabla 1. Dimensiones tratadas en la encuesta y número de preguntas dedicadas a cada área

\begin{tabular}{|c|c|}
\hline Dimensiones & N. ${ }^{\circ}$ de cuestiones \\
\hline $\begin{array}{c}\text { Utilización de materiales y recursos en } \\
\text { materias de metodología AICLE } \\
\text { (dimensión I) }\end{array}$ & 2 \\
\hline $\begin{array}{c}\text { Utilización de la lengua extranjera y } \\
\text { lengua materna (dimensión II) }\end{array}$ & 1 \\
\hline $\begin{array}{c}\text { Empleo de otras metodologías } \\
\text { (cooperativa, aprendizaje basado en } \\
\text { proyectos) (dimensión III) }\end{array}$ & 2 \\
\hline Evaluación (dimensión IV) & 4 \\
\hline Grado de motivación (dimensión V) & 1 \\
\hline Reflexión personal (dimensión VI) & \\
\hline
\end{tabular}

Fuente: Elaboración propia 


\section{Resultados}

Con el fin de delimitar el grado de fiabilidad de los resultados, se evaluó el número de elementos propuestos a partir de los índices de fiabilidad. Para ello, se seleccionó el alfa de Cronbach como método evaluativo de la fiabilidad de dicha encuesta y su resultado fue positivo, pues estuvo cercano a 1 (tabla 2).

Tabla 2. Estadística de fiabilidad

\begin{tabular}{|c|c|}
\hline $\begin{array}{c}\text { Alfa de } \\
\text { Cronbach }\end{array}$ & $\begin{array}{c}\text { Número de } \\
\text { elementos }\end{array}$ \\
\hline 0,973 & 15 \\
\hline
\end{tabular}

Fuente: Elaboración propia

Por otra parte, en cuanto a las dimensiones, se puede decir que tienen ciertas conexiones debido a que forman parte del mismo eje educativo. En la dimensión I dedicada a afirmaciones sobre el uso de materiales y recursos en el estudio de materias pertenecientes al programa de metodología AICLE - se formularon las siguientes premisas $^{4}$, y se obtuvieron los siguientes resultados resumidos en la siguiente tabla:

\footnotetext{
${ }^{4}$ Las afirmaciones elaboradas para esta encuesta han sido adaptadas lingüísticamente al nivel madurativo del alumnado.
} 
Revista Iberoamericana para la Investigación y el Desarrollo Educativo ISSN 2007 - 7467

Tabla 3. Utilización de materiales y recursos en materias de metodología AICLE

\begin{tabular}{|c|c|c|c|c|}
\hline Dimensión I & Nunca & $A$ veces & Generalmente & Siempre \\
\hline $\begin{array}{l}\text { Utilizo el } \\
\text { traductor u } \\
\text { otras } \\
\text { herramientas } \\
\text { en las clases y } \\
\text { tareas AICLE }\end{array}$ & $16 \%$ & $70 \%$ & $14 \%$ & $0 \%$ \\
\hline $\begin{array}{l}\text { Voy a clases } \\
\text { particulares por } \\
\text { dificultades } \\
\text { con las } \\
\text { asignaturas } \\
\text { AICLE }\end{array}$ & $74 \%$ & $12 \%$ & $8 \%$ & $6 \%$ \\
\hline $\begin{array}{l}\text { Los/as } \\
\text { profesores/as } \\
\text { ofrecen } \\
\text { recursos y } \\
\text { estrategias para } \\
\text { las clases } \\
\text { AICLE }\end{array}$ & $22 \%$ & $50 \%$ & $24 \%$ & $4 \%$ \\
\hline $\begin{array}{l}\text { El auxiliar de } \\
\text { conversación } \\
\text { me ayuda con } \\
\text { las clases } \\
\text { AICLE }\end{array}$ & $48 \%$ & $30 \%$ & $18 \%$ & $4 \%$ \\
\hline $\begin{array}{l}\text { El auxiliar de } \\
\text { conversación } \\
\text { trabaja y } \\
\text { explica los } \\
\text { contenidos } \\
\text { AICLE durante } \\
\text { sus horas de } \\
\text { clase }\end{array}$ & $8 \%$ & $42 \%$ & $34 \%$ & $16 \%$ \\
\hline
\end{tabular}

Fuente: Elaboración propia

Sobre la segunda dimensión — referida al empleo de la lengua extranjera y la lengua materna - se formularon dos premisas, de las cuales se obtuvo la siguiente información recogida en esta tabla: 
Tabla 4. Utilización de la lengua extranjera y lengua materna

\begin{tabular}{|c|c|c|c|c|}
\hline Dimensión II & Nunca & A veces & Generalmente & Siempre \\
\hline $\begin{array}{c}\text { Los profesores } \\
\text { hablan en } \\
\text { español en } \\
\text { asignaturas } \\
\text { AICLE }\end{array}$ & $0 \%$ & $86 \%$ & $14 \%$ & $0 \%$ \\
\hline $\begin{array}{c}\text { Me cuesta } \\
\text { seguir las } \\
\text { clases AICLE } \\
\text { por el nivel de } \\
\text { inglés }\end{array}$ & $52 \%$ & $40 \%$ & $8 \%$ & $0 \%$ \\
\hline
\end{tabular}

Fuente: Elaboración propia

La tercera dimensión — dedicada al empleo de otras metodologías - no ha sido estudiada en profundidad debido a la gran cantidad de elementos que se trataron de abordar en este estudio. No obstante, se procuró determinar si las metodologías de aprendizaje cooperativo y aprendizaje basado en proyectos eran empleadas en el proceso de aprendizaje mediante la metodología AICLE. Los resultados obtenidos se resumen en esta tabla:

Tabla 5. Empleo de otras metodologías

\begin{tabular}{|c|c|c|c|c|}
\hline Dimensión III & Nunca & A veces & Generalmente & Siempre \\
\hline $\begin{array}{c}\text { Trabajo más } \\
\text { con mis } \\
\text { compañeros en } \\
\text { AICLE que en } \\
\text { lengua materna } \\
\begin{array}{c}\text { a través de } \\
\text { proyectos y de } \\
\text { forma }\end{array}\end{array}$ & $16.3 \%$ & $14.2 \%$ & $40.8 \%$ & $28.6 \%$ \\
cooperativa & & & & \\
\hline
\end{tabular}

Fuente: Elaboración propia 
La evaluación es un proceso fundamental en el aprendizaje que trata de delimitar el grado de adquisición de los conocimientos. En este caso, nos encontramos en un complejo proceso debido al triple foco de la metodología AICLE: lenguas, cognición y contenidos. He aquí los resultados obtenidos en la tabla 6:

Tabla 6. Evaluación

\begin{tabular}{|c|c|c|c|c|}
\hline Dimensión IV & Nunca & A veces & Generalmente & Siempre \\
\hline $\begin{array}{c}\text { La evaluación } \\
\text { de asignaturas } \\
\text { AICLE es más } \\
\text { difícil que en } \\
\text { las asignaturas } \\
\text { no AICLE }\end{array}$ & $18 \%$ & $42 \%$ & $32 \%$ & $4 \%$ \\
\hline $\begin{array}{c}\text { Me evalúan de } \\
\text { diferentes } \\
\text { formas en el } \\
\text { programa } \\
\text { AICLE } \\
\text { (exámenes, } \\
\text { proyectos, } \\
\text { tareas, etc.) }\end{array}$ & $12 \%$ & $36 \%$ & $24 \%$ & $28 \%$ \\
\hline
\end{tabular}

Fuente: Elaboración propia

El grado de motivación en el aprendizaje del alumnado es un elemento indispensable que determina la conducta y actitud del alumno hacia su aprendizaje. Por estos motivos, se ha creado una dimensión para este factor, lo que permitió conseguir la siguiente información recogida en esta tabla: 
Tabla 7. Grado de motivación

\begin{tabular}{|c|c|c|c|c|}
\hline Dimensión V & Nunca & A veces & Generalmente & Siempre \\
\hline $\begin{array}{c}\text { He pensado en } \\
\text { abandonar el } \\
\text { programa } \\
\text { AICLE }\end{array}$ & $60 \%$ & $28 \%$ & $8 \%$ & $4 \%$ \\
\hline $\begin{array}{c}\text { He pensado } \\
\text { que las } \\
\text { asignaturas } \\
\text { AICLE son } \\
\text { más difíciles }\end{array}$ & $32 \%$ & $38 \%$ & $18 \%$ & $12 \%$ \\
\hline $\begin{array}{c}\text { Participo más } \\
\text { en las clases } \\
\text { AICLE que en } \\
\text { las lecciones } \\
\text { de lengua } \\
\text { materna }\end{array}$ & $18 \%$ & $30 \%$ & $32 \%$ & $10 \%$ \\
\hline $\begin{array}{c}\text { Me gustan más } \\
\text { las clases en } \\
\text { lengua materna } \\
\text { que en inglés }\end{array}$ & $32 \%$ & $28 \%$ & $26 \%$ & \\
\hline
\end{tabular}

Fuente: Elaboración propia

Por último, en la dimensión sobre sugerencias de posibles modificaciones del programa bilingüe, los alumnos emitieron respuestas de libre configuración para determinar aquellas áreas de mejora en esta metodología de aprendizaje que se resumen en la siguiente tabla: 
Tabla 8. Reflexión personal

\begin{tabular}{|l|l|}
\hline \multicolumn{1}{|c|}{ Dimensión VI } & \multicolumn{1}{|c|}{ Respuestas de libre configuración } \\
\hline \multirow{2}{*}{ ¿ué cambiarías del programa AICLE? } & $\begin{array}{l}\text { Explicar más detenidamente para } \\
\text { entender mejor. } \\
\text { Cambiaría la elección del auxiliar de } \\
\text { conversación. }\end{array}$ \\
& $\begin{array}{l}\text { No estudiar tanto vocabulario. } \\
\text { Cambiaría que los profesores te dieran } \\
\text { consejos de cómo facilitar el estudio. }\end{array}$ \\
& \begin{tabular}{l} 
El nivel de inglés. \\
\hline
\end{tabular} \\
\hline
\end{tabular}

Fuente: Elaboración propia

\section{Discusión}

En primer lugar, se puede señalar que $70 \%$ de los estudiantes a veces usa herramientas de traducción para seguir las materias no lingüísticas y pertenecientes al programa educativo bilingüe. Asimismo, en la tercera afirmación se observa que $50 \% a$ veces y $22 \%$ nunca reciben recursos y estrategias, tales como métodos o materiales de estudio para sus lecciones de metodología AICLE. Estos resultados sugieren la necesidad de ofrecer más herramientas de aprendizaje.

En cuanto a la figura del auxiliar de conversación, 48 \% del alumnado nunca recibe apoyo de este recurso humano, mientras que $30 \%$ señala que solo a veces cuenta con esa ayuda. Teniendo en cuenta que posteriormente $42 \%$ indicó que $a$ veces trabajaba los contenidos de la materia perteneciente al programa de metodología AICLE, se debe profundizar en los motivos de esta situación.

Por otra parte, en la dimensión dedicada al uso de las lenguas extranjera y materna, $86 \%$ señaló que $a$ veces el profesorado utilizaba la lengua materna en asignaturas AICLE. En tal sentido, el alumnado argumentó que los docentes la empleaban para resolver conflictos o para hacer comprender contenidos de mayor complejidad. En una metodología AICLE se debe tener en cuenta que si bien la lengua materna es un vehículo 
de comunicación esencial para el aprendizaje, también se debe favorecer la utilización de la lengua extranjera para promover la competencia comunicativa de los aprendices en un nuevo idioma, por lo que ambas deben complementarse para conseguir la optimización del aprendizaje.

En la tercera dimensión, $40.8 \%$ señaló que generalmente empleaban metodologías cooperativas y basadas en proyectos, mientras que $28.6 \%$ comentó que siempre lo hacían. Asimismo, reconocieron que en la asignatura Geografía e Historia realizaban más proyectos de forma cooperativa. Además, en sus respuestas de justificación indicaron que preferían estas metodologías porque sentían que habían aprendido más mientras la pasaban bien con sus compañeros. Al respecto, Pastor Martínez (2011) considera que la combinación de la metodología AICLE con metodologías cooperativas es indispensable para la formulación de comunidades de aprendizaje mientras adquieren los valores y las normas de la comunidad. A pesar de que se requiere mayor profundización en estas metodologías, estos resultados demuestran ciertas preferencias por las metodologías cooperativas y basadas en proyectos.

En la dimensión dedicada a la evaluación de las asignaturas, 42 \% consideró que a veces les parecía más difícil, mientras que $32 \%$ opinó que generalmente. Aunque la evaluación se debe realizar a partir de la normativa vigente, los currículos actuales en la comunidad canaria no adquieren esta triple perspectiva en esta metodología de aprendizaje de lenguas, contenidos y cognición. Por tanto, la realización de la evaluación, incluso desde la perspectiva del profesorado, es una labor de gran complejidad debido a la incompatibilidad de los objetivos de la metodología AICLE con la normativa educativa vigente. Asimismo, se observa positivamente que el profesorado siempre (28\%) y generalmente (24\%) emplea numerosos productos de aprendizaje. A pesar de que se trata de un método evaluativo, este resultado evidencia una percepción favorable de los estudiantes.

Sobre la dimensión motivacional del alumnado, muy poco alumnado se ha planteado de forma genérica — generalmente $(8 \%)$ - o continua — siempre $(4 \%)$ abandonar el programa de educación bilingüe. Sin embargo, más de un cuarto del alumnado se plantea tal decisión (28\%) en algunas ocasiones, lo que demuestra la necesidad de determinar aquellas áreas en las que puedan demostrar mayor debilidad y buscar métodos y recursos para su fortalecimiento.

Por otro lado, $26 \%$ y $28 \%$ del alumnado consideró que a veces y generalmente, respectivamente, le gustan más las clases en lengua materna que en inglés. Debido a ello, se debe profundizar en el componente motivacional de la estrategia, así como en las 


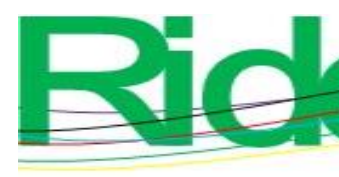

Revista Iberoamericana para la Investigación y el Desarrollo Educativo ISSN 2007 - 7467

razones que sustentan esos porcentajes. Al respecto, se ofrecen algunas opiniones de los estudiantes:

- "Porque tener que estudiar en inglés es más difícil".

- "Porque en español las clases las entiendo mejor y me puedo explicar con más soltura porque es mi lengua materna".

- "Por el vocabulario".

Estos comentarios sobre sus experiencias con la metodología AICLE suscitan ciertos problemas en la comprensión de la lengua extranjera. Entre ellos, se hace referencia en múltiples ocasiones al vocabulario como un impedimento para el aprendizaje. A pesar de que un programa educativo de modalidad bilingüe puede favorecer el aprendizaje de un vocabulario receptivo (Mora Ramos, 2014), este podría llegar a constituirse como un hándicap en el proceso de aprendizaje en la metodología AICLE (Castellano-Risco, 2017), dependiendo de los métodos usados para su tratamiento y aprendizaje en el aula. Asimismo, se observa que el alumnado reconoce que estudiar en inglés es más complicado porque no es su lengua materna. Esta afirmación sugiere el reforzamiento de recursos y técnicas de estudio para la aplicación de la metodología AICLE, pues esos estudiantes proceden de centros de educación primaria en los que se ha aplicado dicha misma metodología.

La última dimensión —dedicada a un ejercicio de autoconciencia sobre el programa educativo - evidencia diversas áreas que el alumnado considera que se deben reforzar (p. ej., el vocabulario receptivo, la figura del auxiliar de conversación y la adaptación de la lengua) para optimizar la aplicación de la metodología AICLE en el aula.

\section{Conclusiones}

Una de las principales conclusiones que se pueden reseñar luego de culminar este trabajo de investigación tiene que ver con la necesidad de fortalecer ciertas dimensiones de la metodología AICLE relacionadas con el grado motivacional, la figura del auxiliar de conversación y la implementación de estrategias y recursos de apoyo, elementos esenciales para impulsar la formación en una lengua extranjera.

Además, se debe decir que la aplicación un cuestionario como el usado en esta indagación supuso para el estudiante un medio de autorreflexión que favoreció el tercer enfoque educativo, es decir, la cognición en la metodología AICLE. En otras palabras, esta tipología de investigaciones le ofrece al alumno la posibilidad de evaluar no solo las estrategias didácticas usadas en el aula, sino también su competencia comunicativa y su 
motivación, así como la necesidad de contar con recursos y apoyos para consolidar su proceso formativo. De este modo, el aprendiz estudia los contenidos, la lengua y al propio individuo a través de un proceso de objetivos simultáneos.

\section{Futuras líneas de investigación}

Finalmente, se pueden mencionar nuevas líneas de investigación, las cuales podrían incluir variables como el desarrollo académico del alumnado durante toda la educación secundaria con el fin de observar la evolución de los parámetros seleccionados; asimismo, la asimilación de la lengua extranjera como mecanismo dual de aprendizaje y comunicación en el aula durante la etapa de educación secundaria, así como un análisis más detallado sobre las funciones de la figura del auxiliar de conversación, y sobre los métodos estratégicos de aprendizaje empleados por los docentes.

\section{Referencias}

Castellano-Risco, I. (2017). Cómo el uso de un enfoque AICLE puede afectar al vocabulario receptivo en estudiantes de secundaria. En Nieto Caballero, G. (ed.), Nuevas aportaciones al estudio de la enseñanza y aprendizaje de lenguas (pp. 137-149). Extremadura: Servicio de Publicaciones de la Universidad de Extremadura.

Comisión de Europa (2001). Marco Común Europeo de Referencia para las Lenguas: aprendizaje, enseñanza y evaluación. Cambridge: Cambridge University Press.

Coyle, D., Hood, P. and Marsh, D. (2010). CLIL. Content and Language Integrated. Cambridge: Cambridge University Press.

Dalton-Puffer, C., Llinares, A., Lorenzo, F. and Nikula, T. (2014). You can stand under my umbrella. Immersion, CLIL and Bilingual Education. A response to Cenoz, Genesee \& Gorter (2013). Applied Linguistics, 35(2), 213-218.

Durán Martínez, R. (2018). Análisis comparativo de la percepción de los docentes sobre los programas bilingües en la Educación Primaria y Secundaria. Revista Complutense de Educación, 29(3), 865.

Fernández, M., Mena, L. y Riviere, J. (2010). Fracaso y abandono escolar en España. Barcelona: Fundación La Caixa, Colección Estudios Sociales 29. Barcelona: Fundación La Caixa, Colección Estudios Sociales 29. Recuperado de http://multimedia.lacaixa.es/lacaixa/ondemand/obrasocial/pdf/estudiossociales/V ol29_co mpleto_es.pdf 


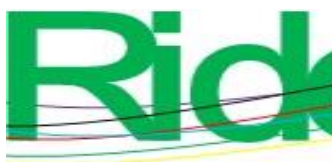

Revista Iberoamericana para la Investigación y el Desarrollo Educativo ISSN $2007-7467$

Martin Frigols, M. J. y Marsh, D. (2014). Informe de evaluación externa: Programa CLIL de la Consejería de Educación, Universidades y Sostenibilidad de Canarias. Recuperado de https://www.consejoescolardecanarias.org/wpcontent/uploads/2015/03/Evaluaci\%c3\%b3n-de-la-implantaci\%c3\%b3n-delprograma-CLIL-en-Canarias-2014.pdf

Julián de Vega, M. (2013). La coordinación docente en modelos AICLE. Padres y Maestros/Journal of Parents and Teachers, (349), 21-24.

Loaiza Villalba, N. L. y Galindo Martínez, A. G. (2014). Estilos de aprendizaje de segundas lenguas y formación bilingüe consecutiva en educación primaria, secundaria y superior: hacia un estado del arte. Lenguaje, 42(2), 291-314.

Mehisto, P. (2012). Criteria for producing CLIL learning material. Encuentro, (21), 1533.

Monarca, H. A., Rappoport, S. y Fernández González, A. F. (2012). Factores condicionantes de las trayectorias escolares en la transición entre enseñanza primaria y secundaria. Revista Española de Orientación y Psicopedagogía, 23(3), $49-62$.

Mora Ramos, I. (2014). Análisis del tamaño del vocabulario receptivo en alumnos de sección bilingüe y no bilingüe de educación primaria. Campo Abierto. Revista de Educación, 33(2), 11-28.

Pastor Martínez, M. R. (2011). CLIL and cooperative learning. Encuentro, 20, 109-118.

Roca, E. (2010). El abandono temprano de la educación y la formación en España. Revista de Educación, (número extraordinario), 31-62.

Rodríguez-Sabiote, C., Madrid, D., Ortega-Martín, J. L. y Hughes, S. P. (2018). Resultados y conclusiones sobre la calidad de los programas AICLE en España. En Ortega Martín, J. L., Hughes, S. y Madrid, D. (eds.), Influencia de la política educativa en la enseñanza bilingüe (pp. 141- 159). Madrid: Ministerio de Educación, Ciencia y Deporte (MECD).

Signoret, A. (2013). Bilingüismo en la infancia. México: Universidad Nacional Autónoma de México. 